\title{
Conocimientos sobre violencia familiar en padres o cuidadores de escolares de un colegio de educación básica'
}

\author{
Eva Chanamé Ampuero² \\ Delia Luzmila Danjoy León³
}

Carolina Cerna ${ }^{4}$

Julio Zurita ${ }^{5}$

Roció Valentín 5

Miriam Roque 5

doi:10.11144/Javeriana.ie19-2.cvfp

Cómo citar: Chanamé Ampuero E, Danjoy León DL, Cerna C, Zurita J, Valentín R, Roque M. Conocimientos sobre violencia familiar en padres o cuidadores de escolares de un colegio de educación básica. Investig Enferm Imagen Desarr. 2017;19(2):29-40. http:// dx.doi.org/10.11144/Javeriana.ie19-2.cvfp

1. Artículo original de investigación. Fecha de recepción: 29 de abril de 2015. Fecha de aceptación: 2 de marzo de 2016.

2. Enfermera. Maestra en Salud Pública y Gestión Sanitaria. Docente asociada, Facultad de Enfermería de la Universidad Peruana Cayetano Heredia (UPCH), Lima, Perú. Research Fellow Program for Advanced Research Capacities for AIDS (PARACAS), Instituto de Medicina Tropical Alexander von Humbolt-UPCH. Correo electrónico: eva.chaname@upch.pe

3. Enfermera. Docente auxiliar, Facultad de Enfermería de la Universidad Peruana Cayetano Heredia, Lima, Perú. Correo electrónico: delia.danjoy@upch.pe

4. Enfermera. Maestra en Administración y Gerencia en Salud. Docente asociada, Facultad de Enfermería, Universidad Peruana Cayetano Heredia, Lima, Perú. Supervisora del Instituto Nacional de Salud del Niño, Lima, Perú. Correo electrónico: carolina.cerna@upch.pe

5. Licenciada, Universidad Peruana Cayetano Heredia. Lima, Perú. Correos electrónicos: juliodsv_1979@hotmail.com,rocio_1889@hotmail.com y franry27@hotmail.com 


\section{Resumen}

Objetivo: Determinar los conocimientos sobre violencia familiar en padres o cuidadores de niños en etapa escolar en una institución educativa nacional del distrito de Ate Vitarte (Lima, Perú). Métodos: Enfoque cuantitativo, tipo descriptivo de corte transversal. Se trabajó con una muestra de 73 padres o cuidadores de escolares de un colegio estatal de Lima. La técnica utilizada fue la encuesta y como instrumento se utilizó un cuestionario que constó de dos partes: la primera sobre datos sociodemográficos de los sujetos de estudio y una segunda parte donde se indaga sobre los conocimientos de violencia familiar en veinte preguntas relativas a violencia física, psicológica, sexual y por negligencia. Resultados: E1 $62 \%$ de los encuestados obtuvo un nivel de conocimiento bajo en violencia física por negligencia; el $52 \%$, en violencia sexual, y el $34 \%$, en violencia psicológica. Por otro lado, se consideró el puntaje global sobre conocimiento de violencia de los cuales el $45 \%$ obtuvo un nivel bajo. Conclusión: Los padres o cuidadores tienen un conocimiento deficiente de los conceptos de violencia y sus tipos.

Palabras clave: violencia doméstica; niño; padres; enfermería en salud comunitaria

\section{Knowledge about Family Violence in Student's Parents or Caregivers of a Basic Education School}

\section{Abstract}

Objective: To determine the knowledge about family violence parents or caregivers of children in school stage have in a state educational institution in Ate Vitarte (Lima, Peru). Methods: Quantitative Approach, descriptive cross- sectional type. The sample was comprised of 73 parents or caregivers of schoolchildren in a state school in Lima. The technique used was surveying and the instrument was a questionnaire which consisted of two parts: the first on socio-demographic data from the Study subjects and a second section was related to the knowledge of family violence in twenty questions on physical, psychological, sexual violence and negligence. Results: $62 \%$ of the respondents got a low score related to knowledge about physical violence by negligence; $52 \%$ in sexual violence, and $34 \%$ in psychological violence. On the other hand, the other score that was taken into account was the overall score on knowledge of violence from which $45 \%$ obtained a low level. Conclusion: Parents or caregivers have poor knowledge of the concepts of violence and their types.

Keywords: domestic violence; boy; parents; community health nursing 


\section{Conhecimentos sobre violência familiar em pais ou cuidadores de escolares de uma escola de ensino básico}

\section{Resumo}

Objetivo: Determinar o nível de conhecimento sobre a violência familiar em pais ou cuidadores de crianças em idade escolar uma instituição de ensino nacional no distrito de Ate (Lima, Perú). Método: Abordagem quantitativa, descritivo transversal ele trabalhou com uma amostra de 73 pais ou responsáveis por alunos de uma escola estadual em Lima. A técnica utilizada foi o instrumento de pesquisa e como um questionário consistia em duas partes: a primeira sobre dados sociodemográficos dos sujeitos do estudo e uma segunda secção investiga o conhecimento da violência familiar em 20 questões relativas à violência física foi utilizado, psicológica, sexual e negligência. Resultados: O $62 \%$ dos inquiridos recebeu um baixo nível de conhecimento em violência física por negligência, o $52 \%$ sobre violência sexual e o $34 \%$ sobre violência psicológica. Por outro lado, a pontuação geral no conhecimento da violência, dos quais $45 \%$ obtido foi considerado baixo. Conclusão: Os pais ou cuidadores têm uma má compreensão dos conceitos de violência e seus tipos.

Palauras-chave: violência doméstica; criança; pais; enfermagem em saúde comunitária 


\section{Introducción}

La familia es una institución social, anterior al ordenamiento jurídico. Está integrada por un conjunto de personas unidas por lazos de parentesco que son vínculos de afinidad o por vínculos de consanguinidad. Precisamente, los fuertes lazos surgidos en la familia-funcionamiento familiar constituyen una base sólida en las relaciones familiares con gran impacto en la sociedad; sin embargo, el relajamiento de estos vínculos determina que sea dentro de la célula familiar donde se manifiestan los primeros síntomas del mal. En ese sentido, hasta hace pocas décadas, el problema de la violencia familiar había sido desdeñado por las sociedades en el mundo y por el aparato estatal de cada sociedad; pero, en la actualidad, se ha producido un cambio radical, dado que los Estados muestran un especial interés por este tema y, en la medida de lo posible, ofrecen un abanico de alternativas de solución, conscientes de que estas manifestaciones de violencia - sean de índole físico, psicológico, sexual e incluso el maltrato sin lesión, con graves consecuencias individuales y colectivas - constituyen un sintoma de distorsiones y manifiestan una grave vulneración a los derechos humanos y libertades y, por ende, un grave obstáculo a los miembros de la familia y de la sociedad en general (1).

La frecuencia de los actos violentos cometidos en el marco de la familia, considerada el conjunto de personas que viven bajo un mismo techo, pone en cuestión el concepto tradicional de la familia como un remanso de paz y tranquilidad. Parece que vivimos en una época en la que todo queda trastocado (2).

El fenómeno de la violencia y el maltrato dentro del ámbito familiar no es un problema reciente. Los análisis históricos revelan que ha sido una característica de la vida familiar tolerada, aceptada desde tiempos remotos. Sin embargo, algunas décadas atrás, expresiones como niños maltratados, mujeres golpeadas o abuso sexual tal vez habrian sido comprendidas, pero no sinónimo de graves problemas sociales (3).

Los problemas intrafamiliares afectan la conducta del niño, sobre todo en una etapa crucial como la escolar, cuando va formando su propia personalidad. Para este caso, Erikson se centra más en los determinantes socioculturales del desarrollo humano: las personas se forman superando ocho tipos de conflictos, que van desde la confianza frente a desconfianza en la infancia hasta la integridad frente a la desesperación en la ancianidad. Propio del conductismo es la consideración del desarrollo humano como condicionado pasivamente por ambiente, que puede ser moldeado por los refuerzos y los castigos (4).

El Movimiento Flora Tristán (2002) reveló que en el Perú seis de cada diez hogares viven con violencia, en una prevalencia del 35\% para la agresión psicológica y del $17 \%$ para la física (5). Estos datos nos indican que, en la actualidad, la violencia familiar continúa siendo un problema de gran dimensión, con repercusiones negativas principalmente en el desarrollo social, y no solo de la víctima, sino de la sociedad en su conjunto, puesto que en el futuro estos niños que viven en ese ambiente de violencia están predispuestos a ser agresores y a generar violencia en sus hogares, lo cual se vuelve así una cadena indefinida. 
En el estudio de Giraldo, Manrique y Torres sobre maltrato familiar en los adolescentes de tercer, cuarto y quinto año de secundaria del Colegio Nacional Clorinda Matto de Turner se mostró que el 64,05\% de adolescentes presenta maltrato físico con empleo de las manos (golpes) por parte de los padres, y el 74,74\%, maltrato psicoemocional, al ser agredidos verbalmente con insultos (6).

En el Perú se reconoce la violencia como un problema de salud pública, tanto así que en el 2009 se encontraron 951.135 casos de violencia familiar y 1025 casos de violencia sexual; en el 2010, 2189 casos de violencia familiar y 977 casos de violencia sexual, y en el 2011, 938.084 casos de violencia familiar y 849 casos de violencia sexual, con niños de ambos sexos atendidos y registrados dentro de los distritos y provincias de Lima. En cuanto a casos nuevos (personas que acuden por primera vez) y casos reincidentes (reincidencia en un nuevo hecho de violencia), cada año se registran alrededor de $100.000(7)$.

En la Vigilancia Epidemiológica Centinela de Violencia Familiar realizada por la Dirección Región Regional de Salud de Lima y en conjunto con el Ministerio de la Mujer y Poblaciones Vulnerables (MIMP) se ha encontrado que la notificación de casos de violencia familiar, agredidos, es mayor en el grupo de edad de 10 a 14 años de edad, con 497 casos de niñas y con 400 casos de niños; en el grupo de 5 a 9 años de edad se reportaron 320 casos de niñas y 390 casos de niños de enero-diciembre del 2010 (8).

Por otro lado, la Encuesta Demográfica y de Salud Familiar 2010 preguntó a las mujeres entrevistadas con hijas e hijos en el hogar sobre la forma de castigo o reprimenda que ejerce el esposo o compañero o ella misma a sus hijas e hijos. La forma de castigo más frecuente que utilizó el padre biológico para reprender o castigar a sus hijas e hijos fue la "verbal" (78,3\%); también mencionaron otras formas como "con golpes o castigos físicos" $(32,2 \%)$ y "prohibiendo algo que les gusta" (27,9\%). Al comparar con el año 2000, se han reducido significativamente las dos primeras formas: 7,5 puntos porcentuales en "reprimenda verbal" y 8 puntos porcentuales en "con golpes o castigos físicos"; sin embargo, ha habido un aumento en 6,1 puntos porcentuales en "prohibir algo que les gusta" (9).

Entre las formas de castigo que utilizó la madre biológica para castigar o reprender a sus hijas e hijos, la reprimenda verbal tuvo mayor incidencia $(76,5 \%)$; asimismo, se mencionaron "prohibiendo algo que les guste" (37,7\%), "con golpes" (36,1\%) y palmadas (12,2\%). Respecto al año 2000 , el castigo de palmadas se ha reducido del 22,5\% al 12,2\%; las otras formas como reprimenda verbal y golpes disminuyeron en 8,5 y 4,7 puntos porcentuales, respectivamente, mientras que el castigo basado en prohibir algo que les gusta aumentó 9,6 puntos porcentuales (9).

La importancia de este problema motivó nuestro interés de estudio, ya que, en la labor de enfermería con la familia y sus miembros, es necesario que el conocimiento de lo que es violencia esté claro para ambos padres, de manera que puedan actuar conociendo qué están realizando. Por ello, contar con información de base que nos permita desarrollar estrategias para controlar la violencia en padres y cuidadores de niños, se evidencia como una 
oportunidad de intervención. Por todo lo anterior, se pretende determinar qué tanto conocimiento sobre violencia familiar tienen padres o cuidadores de niños en etapa escolar.

\section{Método}

Este fue un estudio cuantitativo-descriptivo, porque estuvo dirigido a determinar los conocimientos sobre violencia familiar que poseen padres o cuidadores de niños en etapa escolar, y fue de corte transversal, porque se recolectó la información en un solo momento, haciendo el corte en un tiempo. La investigación se realizó en una institución educativa nacional del distrito de Ate Vitarte (Lima, Perú), donde se brinda educación primaria y secundaria. La población de estudio estuvo conformada por 185 padres o cuidadores de los niños en etapa escolar de primaria; mientras que la muestra fue de 73 padres seleccionados por muestreo aleatorio simple. La fórmula fue:

$$
\begin{aligned}
& \mathrm{n}=\frac{(1,96)^{2}(0,95)(0,05)}{(0,05)^{2}} \\
& \mathrm{n}=72,990
\end{aligned}
$$

La técnica utilizada fue la encuesta, y como instrumento se utilizó un cuestionario que constó de dos partes: la primera, sobre datos sociodemográficos de los sujetos de estudio; la segunda, donde se indagó sobre los conocimientos de violencia familiar a partir de veinte preguntas. Los reactivos fueron cerrados de opción múltiple sobre violencia familiar física, psicológica, sexual y por negligencia, cada una con cuatro alternativas de respuesta y una sola correcta. Clasificamos las preguntas por categoría:

Violencia física:

- ¿Qué es violencia física?

- ¿ ¿Usted cree que los castigos drásticos son formas de corregir al niño?

Violencia psicológica:

- ¿Qué es violencia psicológica?

- ¿Qué problemas sociales son predisponentes para la violencia familiar?

- ¿Cuáles son las repercusiones en los niños que son testigos de violencia familiar?

Violencia sexual:

- ¿Qué es violencia sexual?

- ¿Cuál es la consecuencia del abuso sexual dentro de la familia?

Negligencia:

- ¿Qué es violencia por abandono o negligencia?

- Los tipos de violencia mencionados anteriormente. ¿Qué tipo de violencia es cuando una madre, en el momento en que su hijo no quiere 
comer, no insiste en que coma, mencionándole que si tiene hambre no le pida, que no le va dar nada de comer por mañoso y lo deja sin cenar?

Violencia familiar:

- Para usted ¿qué es violencia familiar?

- ¿Cuál de las siguientes situaciones aumenta la posibilidad de que se presente un caso de violencia familiar?

- ¿Cuáles son las principales manifestaciones de violencia familiar?

- La violencia familiar no se da de un momento a otro, es un proceso de aumento de muchos factores, es decir. Ciclos. ¿Cuál es el ciclo de la violencia familiar?

- Como consecuencia de la violencia familiar el niño puede presentar:

- ¿Cuáles son las causas de la violencia familiar?

Entre otras preguntas generales.

Se asignó a cada pregunta un puntaje de 1 si es correcta y de 0 si es incorrecta, para un total de 20 puntos, que se clasificaron aplicando la Escala de Estaninos: bajo $=0-6$, medio $=7-12$ y alto $=13-20$. La valide $z$ del instrumento fue de $p=0,000$ y su confiabilidad de Richardson F20 = 0,69.

El proyecto contó con la autorización del colegio y la aprobación del Comité Institucional de Ética para Humanos de la Universidad Peruana Cayetano Heredia (código 59828 del 9 de octubre de 2012). Este Comité está registrado en la Office for Human Research Protections, con códigos, institucional y de la Clasificación Internacional de Enfermedades: ORG0000671 y IRB00001014, respectivamente. Tiene el código de garantía federal (Federalwide Assurance) FWA00000525 y está registrado por el Instituto Nacional de Salud del Perú con el código CEI-14.

Con las autorizaciones respectivas, se reunió a los padres de familia en una de las aulas asignadas por la dirección del colegio para tal fin. Se optó por el horario de la tarde, por ser más tranquilo y por la disponibilidad de aulas. Una vez reunidos los padres o cuidadores en el aula, se les explicó la naturaleza del estudio, se les solicitó la firma del consentimiento informado y de participación y se les entregó el cuestionario. El instrumento se aplicó a padres o cuidadores de niños de cuarto, quinto y sexto grado de primaria, en diciembre de 2012. La tabulación de datos se llevó a cabo en Microsoft Excel. El procesamiento de la información se realizó en el software estadístico Statistical Package for the Social Science (SPSS). El análisis de datos se realizó de acuerdo con los objetivos estipulados en el estudio, elaborando cuadros estadísticos de frecuencia y medida de resumen (porcentaje). Los resultados se presentan en tablas de doble entrada.

Debido a que por la investigación realizada y, posteriormente, por la aplicación del instrumento nos dimos cuenta de que es un tema de responsabilidad social del cual depende la salud física y mental de los escolares, se consideró la necesidad de capacitar a los padres o cuidadores; además, se incluyó a los docentes, quienes son un ente importante en la difusión de la protección del menor contra la violencia familiar. 


\section{Resultados}

En la tabla 1, del total de los encuestados, el $62 \%$ obtuvo un nivel de conocimiento bajo sobre violencia física, y el 33\%, un nivel de conocimiento medio. Asimismo, solo un $5 \%$ presentó un nivel de conocimiento alto.

TABLA 1. Nivel de conocimiento sobre violencia física en padres o cuidadores en niños en etapa escolar, 2012

\begin{tabular}{|l|r|r|}
\hline \multicolumn{1}{|c|}{ Conocimiento violencia fisica } & \multicolumn{1}{|c|}{$\mathbf{n = 7 3}$} & \multicolumn{1}{|c|}{ \% } \\
\hline Bajo & 45 & 62 \\
\hline Medio & 24 & 33 \\
\hline Alto & 4 & 5 \\
\hline
\end{tabular}

Fuente: resultados de la investigación

La tabla 2 muestra que el $32 \%$ obtuvo un nivel de conocimiento bajo sobre violencia psicológica; mientras que el nivel medio y el nivel de conocimiento alto lo obtuvo un 34\%. En la tabla 3, el 52\% obtuvo un nivel de conocimiento bajo sobre violencia sexual, y el $32 \%$, un nivel de conocimiento medio. Asimismo, el $16 \%$ obtuvo un nivel de conocimiento alto.

TABLA 2. Nivel de conocimiento sobre violencia psicológica, 2012

\begin{tabular}{|l|r|r|}
\hline \multicolumn{1}{|c|}{ Conocimiento violencia psicológica } & $\mathbf{n}=\mathbf{7 3}$ & \multicolumn{1}{c|}{$\%$} \\
\hline Bajo & 23 & 32 \\
\hline Medio & 25 & 34 \\
\hline Alto & 25 & 34 \\
\hline
\end{tabular}

Fuente: resultados de la investigación

TABLA 3. Nivel de conocimiento sobre violencia sexual, 2012

\begin{tabular}{|l|r|r|}
\hline \multicolumn{1}{|c|}{ Conocimiento violencia sexual } & $\mathbf{n}=\mathbf{7 3}$ & \multicolumn{1}{c|}{$\%$} \\
\hline Bajo & 38 & 52 \\
\hline Medio & 23 & 32 \\
\hline Alto & 12 & 16 \\
\hline
\end{tabular}

Fuente: resultados de la investigación

En la tabla 4, del total de los encuestados, el $64 \%$ obtuvo un nivel de conocimiento bajo sobre violencia por negligencia o abandono; el 29\%, un nivel de conocimiento medio. Asimismo, el $7 \%$ obtuvo un nivel de conocimiento alto. La tabla 5 muestra el nivel de conocimiento global sobre violencia. Se muestra que el $45 \%$ obtuvo un nivel de conocimiento bajo, y el $29 \%$, un medio. Asimismo, el $26 \%$ de los cuidadores obtuvo un nivel de conocimiento alto.

TABLA 4. Nivel de conocimiento sobre violencia por negligencia o abandono, 2012

\begin{tabular}{|l|r|r|}
\hline \multicolumn{1}{|c|}{ Conocimiento violencia por negligencia o abandono } & $\mathbf{n}=\mathbf{7 3}$ & \multicolumn{1}{c|}{$\%$} \\
\hline Bajo & 47 & 64 \\
\hline Medio & 21 & 29 \\
\hline Alto & 5 & 7 \\
\hline
\end{tabular}

Fuente: resultados de la investigación 
TABLA 5. Nivel de conocimiento sobre violencia familiar, 2012

\begin{tabular}{|l|r|r|}
\hline \multicolumn{1}{|c|}{ Conocimiento violencia familiar } & $\mathbf{n = 7 3}$ & \multicolumn{1}{c|}{$\%$} \\
\hline Bajo & 33 & 45 \\
\hline Medio & 21 & 29 \\
\hline Alto & 19 & 26 \\
\hline
\end{tabular}

Fuente: resultados de la investigación

Se identificaron como características personales de los cuidadores que en el $52 \%$ de los casos son generalmente las madres quienes están a cargo del cuidado del niño y que, en su gran mayoria, el 66\% de ellas se encuentran en edades comprendidas de los 20 a los 40 años. Asimismo, se evidencia que el $37 \%$ y el $30 \%$ son casadas y convivientes, respectivamente, y que el $73 \%$ provienen de Lima.

\section{Discusión}

El conocimiento es un proceso en virtud del cual la realidad se refleja y reproduce en el pensamiento humano; dicho proceso está condicionado por el proceso del devenir social y se halla unido a la actividad práctica (10).

Con respecto al nivel de conocimientos, en nuestra investigación se encontraron porcentajes altos, sobre todo en el nivel bajo. En cuanto al tema de la violencia física, se identificó un $62 \%$. En relación con la violencia psicológica, se obtuvo un $32 \%$. El 52\% lo obtuvo el tema de violencia sexual. En violencia por negligencia o abandono se obtuvo un $64 \%$, con nivel bajo, y un $45 \%$ obtuvo un nivel de conocimiento bajo sobre la violencia en general. Probablemente sea por esto que el MIMP tiene como misiones: regir políticas públicas bajo la equidad de género, protección y desarrollo social de poblaciones vulnerables y que sufren exclusión; mejorar su calidad de vida, y promover su realización personal y social. Bajo el programa de atención integral a víctimas de violencia familiar y sexual que realizó una revisión de casos en niños menores de 17 años de edad, en el 2008 se encontraron 2405 casos de violencia familiar y 1445 casos de violencia sexual; en el 2009, 951.135 casos de violencia familiar y 1025 casos de violencia sexual; en el 2010, 2189 casos de violencia familiar y 977 casos de violencia sexual; y en el 2011, 938.084 casos de violencia familiar y 849 casos de violencia sexual, en niños de ambos sexos atendidos y registrados dentro de los distritos y provincias de Lima, en cuanto a casos nuevos (personas que acuden por primera vez) y casos reincidentes (reincidencia en un nuevo hecho de violencia) (7).

Por otro lado, según Unicef Perú, diariamente, miles de niños y niñas en el Perú son maltratados física y psicológicamente por sus padres, madres, parientes, profesores o por cualquier adulto que considere al castigo físico como normal, aceptable y hasta "necesario". Las víctimas de estos casos no aparecen en las estadísticas, salvo que los "castigos" hayan originado graves secuelas o la muerte de quienes recibieron los golpes, y aunque esto ocurre con una frecuencia que sorprende e indigna, las diferentes formas de castigo físico siguen siendo vistas como un método de disciplina y aprendizaje. "La letra con sangre entra" o "Le pego para que aprenda" son dos de los lemas que convierten a esta fórmula atroz de enseñanza en un tema justificado y aceptado socialmente (8). A diferencia con nuestro estudio, Unicef menciona 
que el tipo de violencia que se registra en los menores de 18 años tiene su punto más alto $(71 \%)$ en la violencia psicológica con insultos, gritos, desvalorizaciones o rechazos, amenazas de muerte y amenazas de daño físico. Luego le sigue la violencia física (43,9\%) (11).

En el trabajo con la Dirección Regional de Salud de Lima y en conjunto con el MIMP vienen realizando una Vigilancia Epidemiológica Centinela de Violencia Familiar, aplicada en establecimientos del sector de la salud, y se sustenta en la notificación de casos atendidos y que se aplica en las once regiones del Perú. Se encontró en la notificación de casos de violencia familiar (agredidos) que es mayor en el grupo de edad de 10 a 14 años de edad, con 497 casos de niñas y 400 casos de niños; en el grupo de edad de 5 a 9 años de edad se presentaron 320 casos de niñas y 390 casos de niños, en el periodo enero-diciembre del 2010 (8).

Por estos porcentajes obtenidos, se puede mencionar que, en su gran mayoria, desconocen los conceptos generales de violencia familiar, y sus cuatro tipos: física, psicológica, sexual y por negligencia o abandono. Debido a esto, los resultados muestran una similitud con el estudio de Obeso, Palomino y Vargas (12) en que se evidenció que existe una cultura de maltrato infantil en padres o cuidadores de niños y que aún hay una falta de conocimientos de aspectos sobre el tema. Concluyen los autores que la educación en salud es uno de los pilares más importantes para proporcionar información y sensibilizar a los padres de familia o cuidadores con la finalidad de disminuir la incidencia del maltrato infantil.

Los resultados obtenidos de las características personales de los cuidadores registran que de 73 (100\%) personas encuestadas un 52\% son generalmente las madres que están a cargo del cuidado del niño, y que en su gran mayoría (66\%) se encuentran en edades comprendidas entre los 20 y los 40 años. Asimismo, se evidencia que 27 y 22 están casadas y son convivientes, respectivamente, y que 53 provienen de Lima.

Dado que la violencia es un problema de salud pública en todo el mundo, según la 49 ${ }^{a}$ Resolución de la Asamblea Mundial de la Salud de 1996, se considera que la verdadera prevención, a nivel primario, recae en la intervención con los padres y no con los(as) niños(as) (13). Por otro lado, se sabe que la violencia en un niño se puede identificar rápidamente en la escuela; pero esto solo es posible si el docente es capaz de identificar los signos de alarma que son característicos en un niño que sufre de algún tipo de violencia.

De esta manera, las relaciones entre profesores y estudiantes requerirían un cambio de los roles tradicionales asociados a ambos y un incremento de la comunicación. La violencia escolar se ve favorecida, en el caso de los estudiantes, por la falta de motivación e interés, por problemas de autoestima, por dificultades de comunicación personal, por conductas disruptivas o por fracaso escolar.

En el caso de los profesores, son las relaciones verticales de poder, las metodologias rutinarias, la poca sensibilidad hacia lo relacional y afectivo o las dificultades de comunicación, algunos de los elementos que favorecen la violencia escolar (14). 


\section{Conclusiones}

De acuerdo con los resultados del estudio, es alto el porcentaje de padres/ cuidadores con un nivel bajo de conocimientos sobre violencia familiar. Considerando los diferentes tipos de violencia, se destaca que el conocimiento sobre violencia física, junto con el de violencia por negligencia o abandono, es el que presenta el mayor porcentaje de conocimiento bajo. Entre tanto, el conocimiento sobre violencia sexual es medio. Estos resultados llaman la atención, pues indican los padres/cuidadores disponen de escasa información acerca de los conceptos básicos de violencia familiar, lo que podría, en muchos de los casos, influir en su práctica cotidiana.

\section{Recomendaciones}

A pesar de ser un estudio local, se aprecia la necesidad de educar a los padres/cuidadores de niños de la etapa escolar acerca de la violencia familiar. Es preciso trabajar conjuntamente con los establecimientos de salud para realizar programas de fortalecimiento de la promoción de salud y prevención, haciendo hincapié en los temas de violencia familiar.

\section{Financiamiento}

Ninguno.

\section{Conflictos de interés}

Los autores declaran no tener conflicto de intereses.

\section{Referencias}

1. Hidalgo Ciña KL. Intervención del Ministerio Público y funcionamiento familiar de las víctimas de violencia familiar en el Distrito Judicial de La Libertad, 2008. UCV-Scientia [internet]. 2010;2(1):111-21. Disponible en: http://revistas.ucv.edu.pe/index.php/UCV-SCIENTIA/ article/view/500

2. Valtueña JA. La violencia intrafamiliar: análisis de una epidemia social. OFFARM [internet]. 2007;26(8):26-9. Disponible en: http://apps.elsevier. es/watermark/ctl_servlet?_f=10\&pident_articulo=13109809\&pident_ usuario $=0 \&$ pcontactid $=\&$ pident_revista $=4 \&$ ty $=94 \&$ accion $=$ L\&origen =zonadelectura\&web $=$ www.elsevier.es \&lan $=$ es $\&$ fichero $=4 \mathrm{v} 26 \mathrm{n} 08 \mathrm{a} 13$ 109809pdf001.pdf

3. Deloya Avelar ND, Quintero Ariza GA. Prevención de la violencia familiar con jóvenes de secundaria [internet]. 2008. Disponible en: http:// hdl.handle.net/123456789/7105

4. Riesco González M. Desarrollo social y personalidad: una perspectiva interdisciplinaria [internet]. 2007 [citado 2011 dic 2]. Disponible en: http:// webcache.googleusercontent.com/search?q=cache:ZLvTGSL6oa4J:www. uclm.es/varios/revistas/docenciaeinvestigacion/pdf/numero6/DESARROLLOSOCIALYPERSONALIDAD1. doc $+\& c d=3 \& h l=e s \& c t=c l n k \& g l=p e$

5. Elias C, Patiño AM, Lleó R. Movimiento de mujeres y feminista en Perú [internet]. Madrid: ACSUR-Las Segovias; 2009. Disponible 
en: http://perso.unifr.ch/derechopenal/assets/files/obrasportales/ op_20151108_02.pdf

6. Giraldo Giraldo AM, Manrique Pérez C, Torres Chávez L. Maltrato familiar en los adolescentes [internet]. Tesis de pregrado. Universidad Peruana Cayetano Heredia, Lima, Perú; 2007. Disponible en: https:// www.google.com.co/url?sa $=$ t\&rct $=j \& q=\& e s r c=s \&$ source $=$ web\&cd $=1 \& c$ ad=rja\&uact $=8 \& v e d=0$ ahUKEwjGxaCp5-zPAhXD7iYKHeo9BT8QFgg gMAA\&url=http $\% 3 \mathrm{~A} \% 2 \mathrm{~F} \% 2 \mathrm{Fredin}$. gob.pe\%2Fdocumento\%2F109\&us $\mathrm{g}=\mathrm{AFQjCNHYQuLWn2gOioCnaBcQwusMN8vjKA \& sig2=xIocDYnjfYud}$ 5VliUXVezw\&bvm=bv.136499718,d.eWE

7. Ministerio de la Mujer y Poblaciones Vulnerables. Atención Integral a Víctimas de Violencia Familiar y Sexual [internet]. Lima: MIMP; 2012 [citado 2012 mar 20]. Disponible en: http://www.mimp.gob.pe/index. php?option $=$ com_content $\& v i e w=$ article $\& i d=534 \&$ Itemid $=245$

8. Ministerio de la Mujer y del Desarrollo Social. Programa Nacional contra la Violencia Familiar y Sexual [internet]. Lima: MIMDES; 2011 [citado 2011 oct 20]. Disponible en: http://www.mimp.gob.pe/files/programas_nacionales/pncvfs/vigilancia_epidemiologica_vfs.pdf

9. Ministerio de la Mujer y Poblaciones Vulnerables. Plan Nacional de Acción por la Infancia y la Adolescencia 2010-2021 [internet]. Lima; 2010. Disponible en: https://www.google.com.pe/url?sa=t\&rct=j\&q $=\&$ esrc $=$ s\&source $=$ web $\& c d=2 \& c a d=r j a \& u a c t=8 \& v e d=0$ CCIQFjABah UKEwje28fkgJnIAhWLJB4KHSAgC3o\&url=http \%3A \%2F \%2Fwww. mimp.gob.pe \%2Ffiles \%2Fplanes \%2FPNAIA_2012_2021.doc\&usg=A FQjCNGJQ317BpUefns9WKpCHzaYoOc1Lg\&bvm=bv.103388427,d.dmo

10. Conocimiento. En: Diccionario soviético de filosofía [internet]. Montevideo: Pueblos Unidos; 2001. Disponible en: http://www.filosofia.org/enc/ros/conoc.htm

11. Unicef. Violencia golpe a golpe. En: El sacrificio de los niños [internet]. Lima; p. 4-7. Disponible en: http://www.unicef.org/peru/_files/ notas_prensa/carpetasinformativas/violencia.pdf

12. Obeso YLM, Palomino GS, Vargas MRK. Influencia de un programa educativo en el nivel de conocimiento sobre maltrato infantil en padres o cuidadores de niños de 6 a 9 años del C. E. No 2007 S. M. P. [tesis]. Lima: Universidad Peruana Cayetano Heredia; 2007.

13. Organización Mundial de la Salud. Informe mundial sobre la violencia y la salud [internet]. Washington; 2002 [citado 2011 nov 8]. Disponible en: http://www.paho.org/Spanish/AM/PUB/introduccion.pdf

14. Palomero Pescador JE, Fernández Domínguez MR. La violencia escolar y sus causas. Revista Interuniversitaria de Formación del Profesorado [internet]. 2001;(41):19-38. Disponible en: http://www.aufop.com/aufop/uploaded_files/articulos/1247352775.pdf 